\title{
ypTNM staging after neoadjuvant chemotherapy in the Chinese gastric cancer population: an evaluation on the prognostic value of the AJCC eighth edition cancer staging system
}

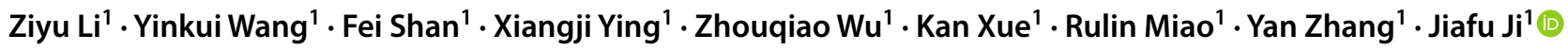

Received: 3 January 2018 / Accepted: 25 April 2018 / Published online: 10 May 2018

(c) The Author(s) 2018

\begin{abstract}
Background This study aims to evaluate the new ypTNM staging system in Chinese gastric cancer patients.

Methods We conducted retrospective survival and regression analyses using a database of gastric cancer patients who underwent neoadjuvant chemotherapy at the Peking University Cancer Hospital and Institute from January 2007 to January 2015. Results A total of 473 patients were included in the study with 28 pathological complete response (pCR) cases, 3 ypT0N1 cases, 65 stage I cases, 126 stage II cases, and 251 stage III cases. The pCR cases had similar survival to stage I patients $(p>0.05)$. The 3-year disease-free survival (DFS) and 5-year overall survival (OS) rates of stage I, II and III patients were significantly different (3-year DFS: 89.0, 75.5, and 39.6\%, $p<0.001$; 5-year OS: 89.6, 65.5, and 36.5\%, $p=0.001$ ). Both ypT and ypN are independent predictors of patient survival, while further log-rank tests showed that the ypN stage is of better prognostic value than ypT. Subgrouping analysis revealed that stage III patients of ypT4b and ypN3 had worse survival compared to the rest of stage III cases $(p<0.001)$. The c-index values of the ypTNM stage and modified ypTNM stage (stage III divided into IIIa and IIIb) were 0.657 and 0.708 , respectively $(p<0.001)$.

Conclusions Our data showed significant differences in survival among gastric cancer patients at different ypTNM stages, indicating its prognostic value in the Chinese population. Further detailed analyses may facilitate the subgrouping of each stage to allow for a more accurate evaluation of disease prognosis in gastric cancer patients.
\end{abstract}

Keywords Stomach cancer $\cdot$ Tumor staging $\cdot$ TNM staging $\cdot$ Neoadjuvant chemotherapy $\cdot$ Chinese population

\section{Introduction}

The TNM staging system is maintained by the Union for International Cancer Control (UICC) and the American Joint Committee on Cancer (AJCC) as a tool for physicians to stage different types of cancer based on the extent of the

Ziyu Li, Yinkui Wang and Fei Shan: Shared first author.

Electronic supplementary material The online version of this article (https://doi.org/10.1007/s10120-018-0830-1) contains supplementary material, which is available to authorized users.

Jiafu Ji

jijiafu@hsc.pku.edu.cn

1 Gastrointestinal Cancer Center, Key Laboratory of Carcinogenesis and Translational Research (Ministry of Education), Peking University Cancer Hospital and Institute, No. 52 Fu-Cheng Road, Hai-Dian District, Beijing 100142, People's Republic of China tumor $(\mathrm{T})$, the involvement of lymph nodes $(\mathrm{N})$, and the presence of metastases (M). Currently, the TNM staging system is globally used for evaluating most malignancies, including gastric cancer. Since it was published in 1976, the TNM staging system remains the most widely implemented resource for cancer staging and served as a baseline for the National Comprehensive Cancer Network (NCCN) guidelines [1]. The previous updates to the TNM staging of gastric cancer were primarily based on patient data collected from the Western populations. However, the release of the eighth edition in 2016 included 25,000 cases of gastric cancer from Eastern and Western countries, thanks to a collaboration with the International Gastric Cancer Association (IGCA) [2].

One major highlight of the eighth edition is the addition of a separate staging system for stomach patients receiving neoadjuvant therapy (e.g., ypTNM staging) [3]. The pretreatment extent of the disease may be clinically (cTNM) or pathologically (pTNM) determined and when neoadjuvant 
therapy is given before surgical intervention, the " $y$ " prefix is added to the TNM category. The concept of y-stage was first described in the third English edition of the Japanese classification of gastric carcinoma [4], yet there were limited data supporting the prognostic value of the ypTNM staging platform. However, the proposed ypTNM staging system was supported by data of 683 patients from the American National Cancer Database (NCDB) [3]. Despite this, further validation of the new ypTNM staging system is not yet available, nor has any data been published about ypTNM staging in the Asian population.

To this end, we performed a retrospective analysis in accordance with the new edition, in the hopes to evaluate the prognostic value of the eighth edition with a special focus on the value of the ypTNM staging system in the Chinese population.

\section{Methods}

\section{Patients}

After obtaining approval from the Peking University Cancer Hospital Ethics Committee, we conducted a retrospective selection of patients who were diagnosed with gastric cancer from January 1, 2007, to January 1, 2015, at the Peking University Cancer Hospital and Institute. The inclusion criteria included: (1) proven diagnosis of gastric adenocarcinoma by endoscopic biopsy prior to surgery; (2) complete clinical and pathological data registered; (3) no signs of distant metastasis prior to the use of chemotherapy; (4) patients that had undergone neoadjuvant chemotherapy prior to surgery; and (5) the D2 gastrectomy was performed in our center. The exclusion criteria included: (1) proven diagnosis with gastrointestinal stromal tumor, lymphoma, neuroendocrine tumor, carcinoid tumors, soft tissue tumors or other non-gastric adenocarcinomas by endoscopic biopsy before surgery; (2) perioperative death within 1 month; (3) patients receiving chemotherapy for other neoplasms within 6 months before the gastric cancer diagnosis; (4) patients receiving neoadjuvant radiotherapy or targeted therapy before surgery; (5) remnant stomach cancer; and (6) patients receiving intraperitoneal chemotherapy or hyperthermic intraperitoneal chemotherapy.

\section{Neoadjuvant chemotherapy}

Our center began implementing neoadjuvant chemotherapy in patients with locally advanced gastric cancer in 2001 . The primary regimen included the selective use of fluorouracil, oxaliplatin, paclitaxel, and other drugs when necessary. The efficacy of neoadjuvant chemotherapy was evaluated every two cycles of treatment using enhanced computed tomography (CT) and ultrasound endoscopy, and the therapy was prematurely terminated in cases of the disease progression. In August 2011, the laparoscopic exploration and rapid cytology of the peritoneal lavage fluid became mandatory. In all resectable cases, elective gastrectomies were scheduled for 4-6 weeks after chemotherapy.

\section{Data collection}

The entry of clinical information on the eligible patients was completed by two independent researchers (Y.K.W. and F.S.) between September 2015 and March 2016 using Epidata v3.1 (EpiData Association, Odense, Denmark). These two investigators discussed and determined patient eligibility and data inconsistencies, while a third researcher was used if an agreement could not be reached.

For this study, the following clinical and pathological data were gathered: age, gender, height, weight, the Eastern Cooperative Oncology Group (ECOG) score, the American Society of Anesthesiologists (ASA) score, family history of cancer, operation time, resection type, multi-organ resection, blood loss, tumor location, T stage, total number of resected lymph nodes, total number of metastatic lymph nodes, pathologic type, differentiation grade, tumor diameter (in long and short axis), and vascular involvement. All included patients were staged according to the eighth AJCC guidelines.

Survival-related data, including overall survival (OS) and disease-free survival (DFS), were also collected. The OS was calculated from the time of the first chemotherapy cycle since diagnosis to the date of death (for any reason) or the most recent follow-up. DFS was determined from the time of the first chemotherapy cycle after diagnosis to any cause of recurrence or metastasis, the date of death if there was no clear recurrence of metastasis, or the last follow-up. The OS and DFS values were recorded in months. The follow-ups were conducted quarterly via telephone, and if the patient (or contact person) could not be reached in three attempts, the patient was considered to be lost.

\section{Statistical analysis}

Data were reported as mean and standard deviation (SD) or frequency and proportion as indicated. Three- and 5-year OS and DFS rates were calculated using the Kaplan-Meier method. We performed log-rank tests to compare the OS and DFS rates across the ypTNM stages, ypT stages, and ypN stages. We conducted univariate Cox regressions, followed by stepwise multivariate Cox models to determine the prognostic value of ypT and ypN stages on the OS and DFS while adjusting for covariates. Variables with $p$ values less than 0.10 in the univariate analyses were included in the multivariate stepwise procedure. A stepwise variable 
selection was based on backward likelihood ratio tests with an entry level of 0.05 and removal of 0.10 .

The current ypTNM staging system does not include ypT0 patients and does not have sub-stages within the ypTNM stage (e.g., Ia or Ib). For this reason, we explored if the current system can be further divided and extended. We divided the patients into 20 groups according to their tumor/node status by combining the five ypT categories $(1 / 2 / 3 / 4 a / 4 b)$ and the four ypN categories $(0 / 1 / 2 / 3)$, from ypT1N0, ypT1N1, and onward to ypT4bN3. We calculated the hazard ratio of each combination compared to the base group within each ypTNM stage (i.e., ypT1N0 for stage I, ypT3N0 for stage II, ypT4aN1 for stage III). From this cluster analysis, we proposed a modified ypTNM staging system. We then used Harrell's $c$-statistic to assess and compare the prediction ability of the current and modified staging systems.

In the overall comparisons, a $p$ value of less than 0.05 was considered as statistically significant. In the pairwise comparisons, the $p$ values were adjusted using the Bonferroni Correction. All reported $p$ values were two-sided. The statistical analysis was performed with SPSS v25 (IBM Corp., Chicago, IL, USA) and Stata v14 (StataCorp, College Station, TX, USA).

\section{Results}

Table 1 displays the clinical, pathological, and surgical characteristics of the eligible patients. A total of 473 patients were included in this study, which encompassed 361 males (76.3\%) and 112 females (23.7\%). The average age was 59 years (26-86 years). The median number of total resected lymph nodes was 30 (15-91), and that of metastatic lymph nodes was two (0-68). The median follow-up duration was 37 months (3-106 months), and 194 patients died at the time of the last follow-up. The 3- and 5-year OS rates were 64.0 and $54.5 \%$, respectively. The 3- and 5-year DFS rates were 59.3 and $51.8 \%$, respectively.

\section{Survival by ypTNM, ypT, and ypN stages}

According to the ypTNM staging system, there were 65 stage I cases, 126 stage II cases, and 251 stage III cases. Figure 1 shows the Kaplan-Meier OS and DFS curves by ypTNM stages. The 3-year OS rates for stage I, II, and III patients were $92.2,77.9$, and $46.0 \%$, respectively (Chi square $=20.7, p<0.001)$. while the DFS rates were 89.0, 75.5 , and $39.6 \%$, respectively (Chi square $=21.9, p<0.001$ ). The 5-year OS rates of stage I, II, III patients were 89.6, 65.5 , and $36.5 \%$, respectively (Chi square $=58.0, p<0.001$ ), while the DFS rates were $84.8,64.5$ and $33.2 \%$, respectively (Chi square $=65.1, p<0.001)$.
The current ypTNM staging system does not include the ypT0 stage. There were $31(6.6 \%)$ ypT0 patients in the current study, of which three were in the ypT0N1 stage and 28 with a pathological complete response (pCR). The three ypT0N1 patients were followed for 42, 61 and 75 months. At the final follow-up, the patients were alive without disease recurrence or metastasis. Log-rank tests showed that neither the OS $(p=0.575)$ nor DFS $(p=0.775)$ rates of the $\mathrm{pCR}$ patients were significantly different from that of the ypTNM stage I patients.

We performed log-rank tests to identify the differences in OS and DFS between adjacent ypT and ypN stage patients. No significant differences were found in the respective pairs of ypT stages, except for ypT4a and ypT4b (Chi square $=42.0, p=0.001<0.0125$ after Bonferroni correction for OS; Chi square $=35.0, p=0.001<0.0125$ after Bonferroni correction for DFS). For adjacent ypN stages, we observed a significant difference on the OS and DFS rates between ypN1 and ypN2 patients (Chi square $=10.9$, $p=0.001<0.017$ after Bonferroni correction for OS; Chi square $=35.0, p=0.001<0.0125$ after Bonferroni correction for DFS), and between ypN2 and ypN3 patients (Chi square $=8.7, p=0.003<0.017$ after Bonferroni correction for OS; Chi square $=12.2, p=0.001<0.017$ after Bonferroni correction for DFS). In Fig. 2, the survival curves of the ypN stages appear to be more diverged than that of the ypT stages (Fig. 3).

\section{ypT and ypN as a significant prognostic factor}

Factors significantly associated with OS and DFS through univariate analysis are shown in Table 2. The following variables remained in the OS prediction model after the stepwise procedure: age, BMI, short tumor diameter, pathological type, ypT stage, ypN stage, and gastrectomy type. Adjusting for the covariates, ypT and ypN stages were significant prognostic factors of OS (both $p<0.001$; Table 3 ). A similar analysis was performed for DFS, and likewise identified that the ypT stage and ypN stage were significantly associated with DFS (both $p<0.001$; Table 3).

\section{Cluster analysis and modified ypTNM grouping}

We performed a cluster analysis and found that patients in stage ypT4b or ypN3 had worse prognostic outcomes than the rest of stage III patients (Supplementary Table 4). No significant hazard ratios were found between stage I and II cancers. Therefore, we divide the stage III patients into two subgroups: group IIIa, including ypT4aN1M0, ypT3N2M0, and ypT4aN2M0; and group IIIb, including ypT2N3M0, ypT4bN0M0, ypT4bN1M0, ypT3N3M0, ypT4bN2M0, ypT4bN3M0, and ypT4aN3M0. Univariate Cox regression found significantly decreased survival for patients in stage 
Table 1 Patient, tumor and treatment characteristics

\begin{tabular}{|c|c|c|}
\hline Characteristics & Mean $\pm \mathrm{SD} / N(\%)$ & Range \\
\hline Age & $59.3 \pm 10.1$ & $26-86$ \\
\hline Female & $112(23.7 \%)$ & \\
\hline Family history (yes) & $85(18 \%)$ & \\
\hline \multicolumn{3}{|l|}{ BMI $\left(\mathrm{kg} / \mathrm{m}^{2}\right)$} \\
\hline$<18.5$ & $27(5.7 \%)$ & \\
\hline $18.5-25.0$ & $304(64.3 \%)$ & \\
\hline $25.0-30.0$ & $128(27.1 \%)$ & \\
\hline$\geq 30.0$ & $14(3 \%)$ & \\
\hline \multicolumn{3}{|l|}{ ECOG score } \\
\hline 0 & $306(64.7 \%)$ & \\
\hline 1 & $146(30.9 \%)$ & \\
\hline 2 & $21(4.4 \%)$ & \\
\hline \multicolumn{3}{|l|}{ ASA score } \\
\hline 1 & $63(13.3 \%)$ & \\
\hline 2 & $329(69.6 \%)$ & \\
\hline 3 & $81(17.1 \%)$ & \\
\hline \multicolumn{3}{|l|}{ Tumor location } \\
\hline Upper stomach & $173(36.6 \%)$ & \\
\hline Middle stomach & $61(12.9 \%)$ & \\
\hline Lower stomach & $209(44.2 \%)$ & \\
\hline Whole stomach & $30(6.3 \%)$ & \\
\hline Diameter in short axis $(\mathrm{cm})$ & $2.9 \pm 2.4$ & $0-19$ \\
\hline Diameter in long axis $(\mathrm{cm})$ & $4.2 \pm 3.2$ & $0-20$ \\
\hline \multicolumn{3}{|l|}{ Pathological type } \\
\hline Adenocarcinoma or adenocarcinoma with NET & $372(78.6 \%)$ & \\
\hline $\begin{array}{l}\text { Signet ring cell carcinoma or adenocarcinoma with signet } \\
\text { ring }\end{array}$ & $8(1.7 \%)$ & \\
\hline Mucinous adenocarcinoma* & $59(12.5 \%)$ & \\
\hline \multicolumn{3}{|l|}{ Differentiation } \\
\hline High & $36(7.6 \%)$ & \\
\hline Middle & $102(21.6 \%)$ & \\
\hline Low & $334(70.8 \%)$ & \\
\hline Vascular cancer embolus (yes) & $158(33.4 \%)$ & \\
\hline \multicolumn{3}{|l|}{ ypT stage } \\
\hline T0 & $31(6.6 \%)$ & \\
\hline $\mathrm{T} 1$ & $33(7.0 \%)$ & \\
\hline $\mathrm{T} 2$ & $67(14.2 \%)$ & \\
\hline T3 & $66(14 \%)$ & \\
\hline $\mathrm{T} 4 \mathrm{a}$ & $246(52.0 \%)$ & \\
\hline $\mathrm{T} 4 \mathrm{~b}$ & $30(6.3 \%)$ & \\
\hline \multicolumn{3}{|l|}{ ypN stage } \\
\hline N0 & $176(37.2 \%)$ & \\
\hline N1 & $98(20.7 \%)$ & \\
\hline $\mathrm{N} 2$ & $85(18 \%)$ & \\
\hline $\mathrm{N} 3$ & $114(24.1 \%)$ & \\
\hline Postoperative stay (day) & $14.6 \pm 17.0$ & $5-283$ \\
\hline Surgery duration (min) & $210.6 \pm 63.5$ & $83-569$ \\
\hline Blood loss (ml) & $171.8 \pm 316.4$ & $50-6500$ \\
\hline \multicolumn{3}{|l|}{ Gastrectomy type } \\
\hline Proximal & $52(11.0 \%)$ & \\
\hline Distal & $186(39.3 \%)$ & \\
\hline
\end{tabular}


Table 1 (continued)

\begin{tabular}{llr}
\hline Characteristics & Mean \pm SD/N $(\%)$ & Range \\
\hline Total & $220(46.5 \%)$ & \\
Thoratic-abdominal joint approach & $15(3.2 \%)$ & \\
Multi-organ excision (yes) & $38(8 \%)$ & $15-91$ \\
Number of resected lymph nodes & $32.1 \pm 11.9$ & $0-68$ \\
Number of metastatic lymph nodes & $4.9 \pm 7.9$ & \\
\hline
\end{tabular}

$B M I$ body mass index, ASA American Society of Anesthesiologists, ECOG Eastern Clinical Oncology Group, NET neuroendocrine tumor, SOX S-1 + oxaliplatin, CapeOX capecitabine + oxaliplatin, FOLFOX $5-\mathrm{FU}+$ leucovorin + oxaliplatin

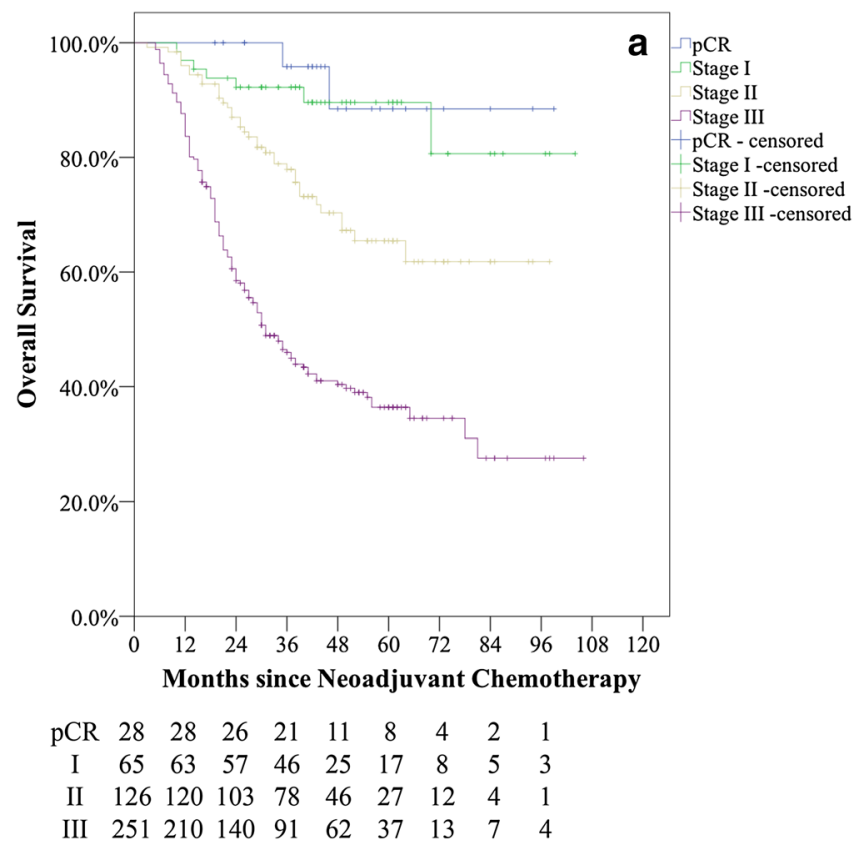

Fig. 1 Post-neoadjuvant chemotherapy stage (ypTNM) and survival, stratified by ypStage groupings. a Overall survival (OS). The 5-year OS of pCR, stage I, II and III patients were 88.5, 89.6, 65.5, and $36.5 \%$, respectively. No significant difference was determined between $\mathrm{pCR}$ and stage I patients $(p=0.514)$. Log-rank analysis was carried out in stage I, II and III cases, and significant differences in OS were determined ( $p<0.001$, respectively). b Disease free survival

IIIb than those in IIIa, with regard to OS (HR 2.62, 95\% CI $1.86-3.67, p<0.001$ ) and DFS (HR 2.62, 95\% CI 1.89-3.62, $p<0.001$ ), which was also confirmed in the multivariate analysis when adjusted for the covariates listed in Table 3 (HR 2.24, 95\% CI 1.57-3.21, $p<0.001$ for OS; HR 2.30, $95 \%$ CI $1.62-3.26, p<0.001$ for DFS).

Next, we assessed the discrimination ability of the current and modified ypTNM (i.e., stage III divided into IIIa and IIIb) staging systems. The c-index of the modified ypTNM staging system, calculated from a univariate Cox model of OS, was significantly higher than that of the current ypTNM system ( $c$-index $=0.708$ vs. $0.657, p<0.001$ ). Similar results were reached using the multivariate Cox

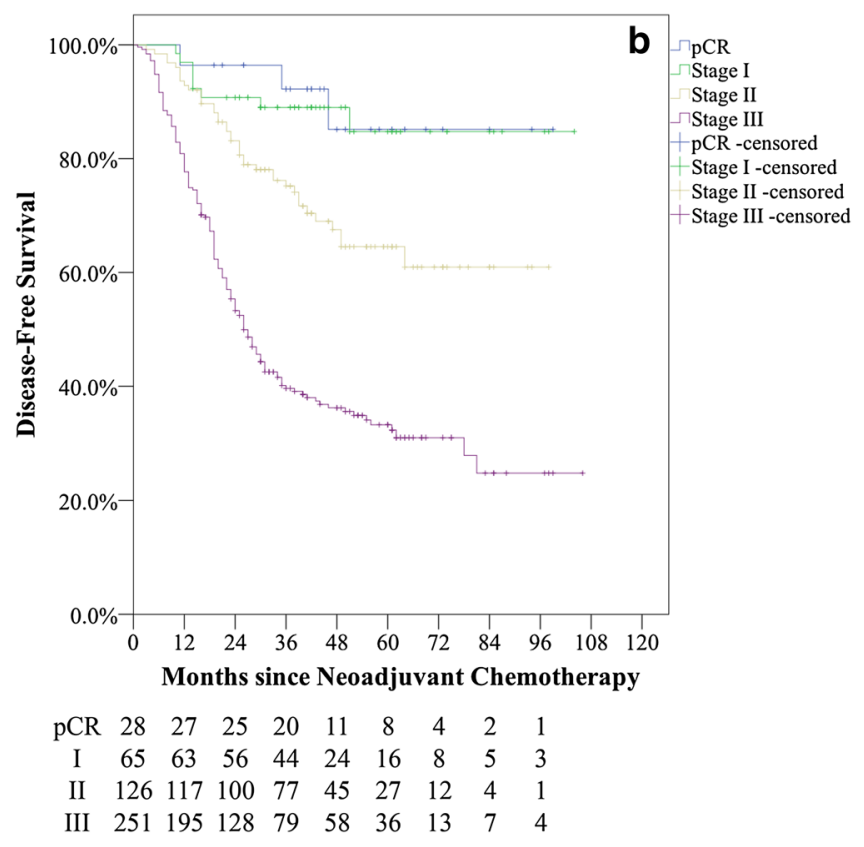

(DFS). The 3-year DFS of pCR, stage I, II and III patients were 92.2, $89.0,75.5$, and $39.6 \%$, respectively. No significant difference was determined between pCR and stage I patients $(p=0.505)$. Log-rank analysis was carried out in stage I, II and III cases, and significant differences in DFS were determined in each comparison $(p<0.001$, respectively). Statistical significance was defined as $p<0.017$ after Bonferroni adjustment

models adjusting for variables in Table 3 ( $c$-index $=0.758$ vs. $0.737 p=0.005$ ). Considering pCR and ypT0N1 were not included in the ypTNM staging system, we included ypT0 into stage I. After combining pCR and stage I patients, the $c$-index of the ypTNM staging (pCR and stages I/II/III) system was 0.681 and that of the modified ypTNM staging (pCR and stages I/II/IIIa/IIIb) system was 0.727 . The difference of 0.046 was likewise significant $(p<0.001)$. 

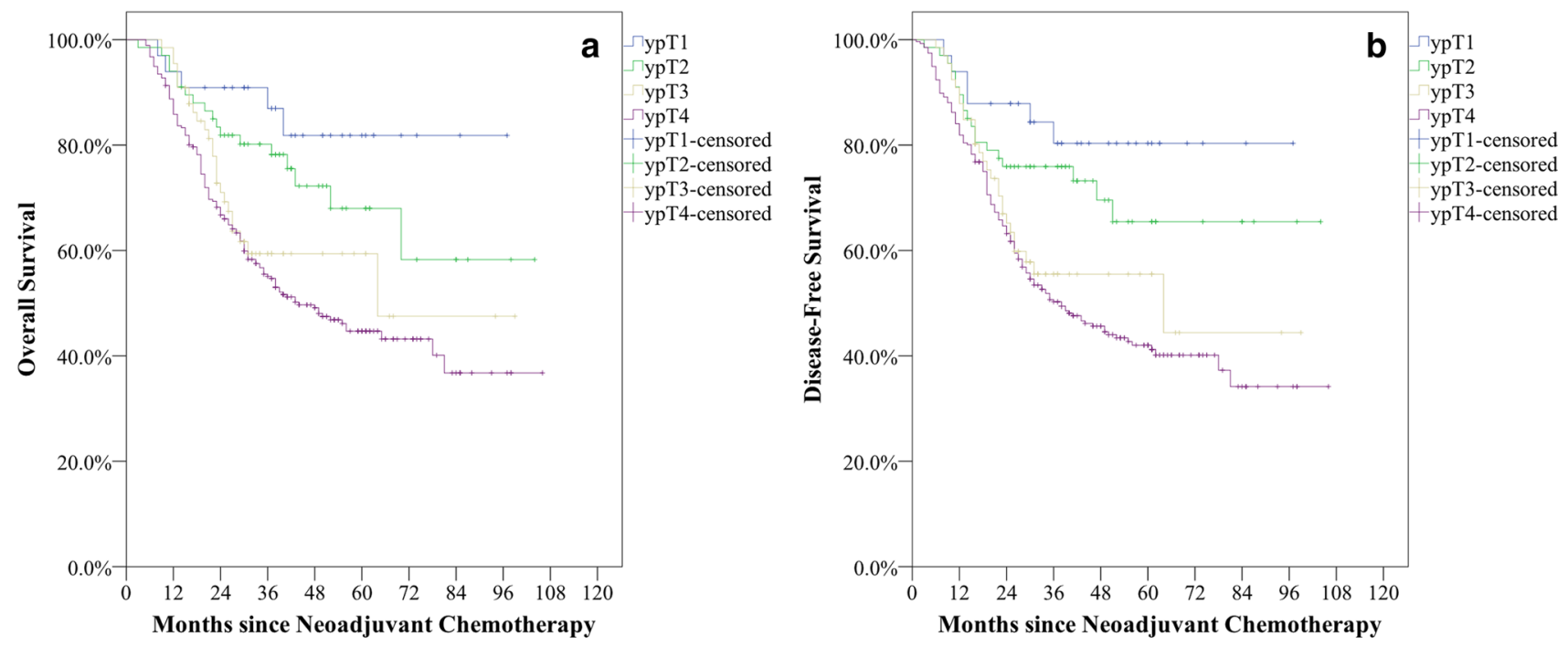
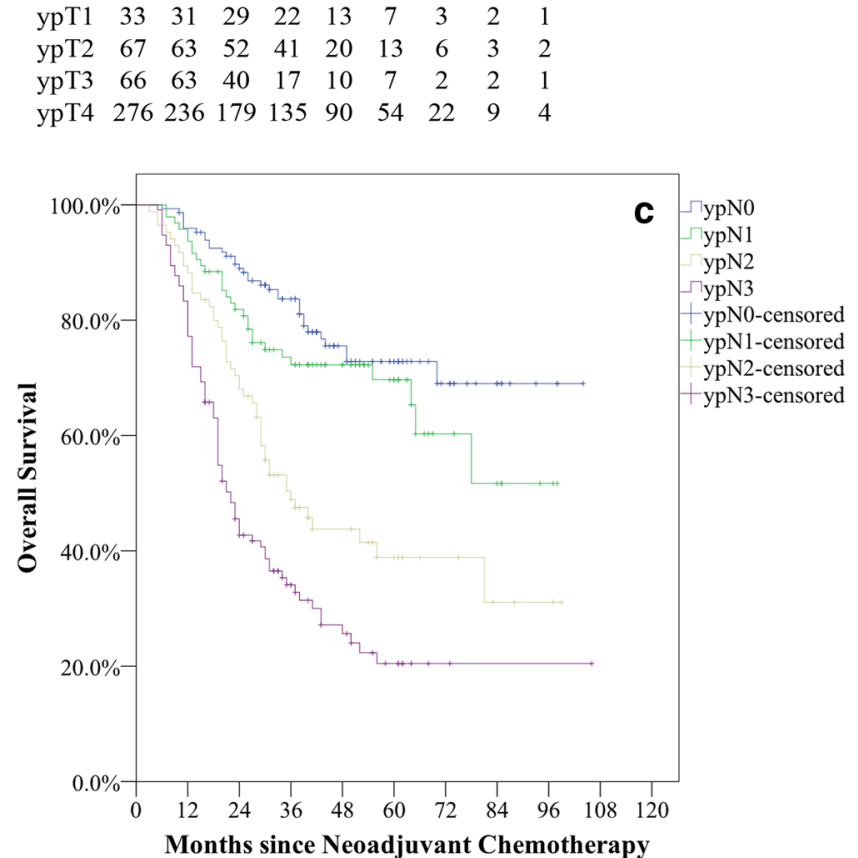

ypN0 $148 \begin{array}{lllllllll}141 & 125 & 100 & 56 & 34 & 16 & 7 & 3\end{array}$

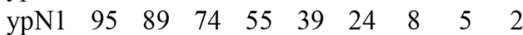

$\begin{array}{llllllllll}\text { ypN2 } & 85 & 75 & 57 & 34 & 21 & 13 & 7 & 3 & 2\end{array}$

$\begin{array}{llllllllll}y p N 3 & 114 & 88 & 44 & 26 & 17 & 10 & 2 & 1 & 1\end{array}$

Fig. 2 Post-neoadjuvant chemotherapy stage (ypTNM) and survival, stratified by ypT or ypN groupings. Statistical analysis (log-rank comparison) was performed in each pair of the adjacent groups. a Overall survival (OS) of respective ypT stage patients. No statistical significant difference was found between the adjacent groups (ypT1 vs. ypT2, $p=0.205$; ypT2 vs. ypT3, $p=0.073$; ypT3 vs. ypT4, $p=0.303$ ). b Disease free survival (DFS) of respective ypT stage patients. No statistical significant difference was found between the adjacent groups (ypT1 vs. ypT2, $p=0.271$; ypT2 vs. ypT3, $p=0.077$; $\begin{array}{lccccccccc}\text { ypT1 } & 33 & 31 & 28 & 20 & 12 & 7 & 3 & 2 & 1 \\ \text { ypT2 } & 67 & 60 & 48 & 39 & 19 & 12 & 6 & 3 & 2 \\ \text { ypT3 } & 66 & 58 & 37 & 16 & 10 & 7 & 2 & 2 & 1 \\ \text { ypT4 } & 276 & 226 & 171 & 125 & 86 & 53 & 22 & 9 & 4\end{array}$

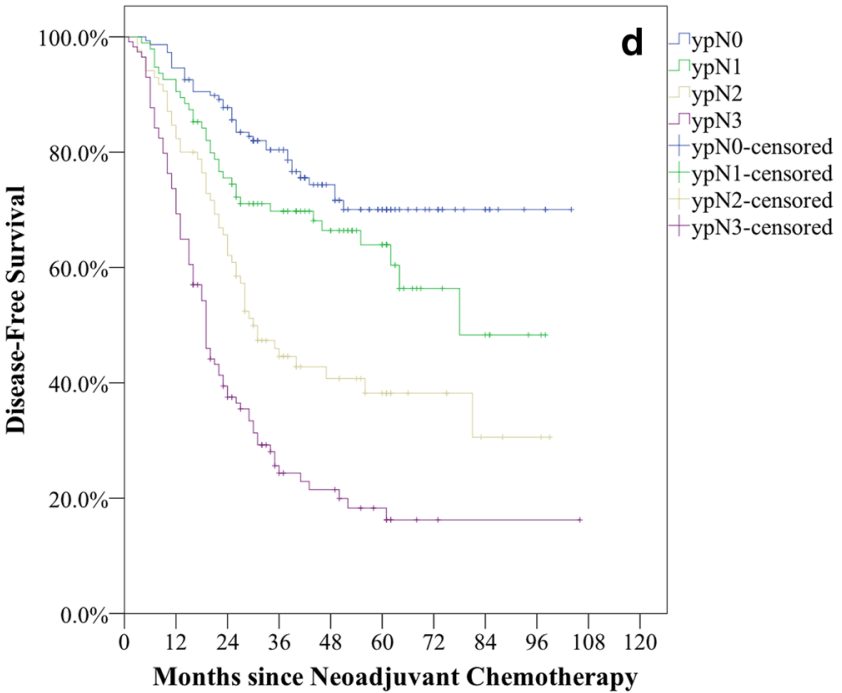

ypN0 $148 \begin{array}{lllllllll}140 & 124 & 97 & 55 & 33 & 16 & 7 & 3\end{array}$

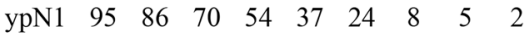

$\begin{array}{llllllllll}\text { ypN2 } & 85 & 70 & 52 & 31 & 20 & 13 & 7 & 3 & 2\end{array}$

$\begin{array}{llllllllll}\mathrm{ypN} 3 & 114 & 79 & 38 & 18 & 15 & 9 & 2 & 1 & 1\end{array}$

ypT3 vs. ypT4, $p=0.361$ ). $\mathrm{c}$ OS of respective ypNstage patients. No statistical significant difference was found between ypN0 and ypN1 $(p=0.080)$, significant differences were determined between ypN1 and ypN2 $(p=0.001)$, and between ypN2 and ypN3 $(p=0.004)$. d DFS of respective ypN stage patients. No statistical significant difference was found between ypN0 and ypN1 $\left(p=0.036^{*}\right)$, significant differences were determined between ypN1 and ypN2 $(p=0.002)$, and between ypN2 and ypN3 $(p=0.001)$. *Statistical significance was defined as $p<0.017$ after Bonferroni adjustment 


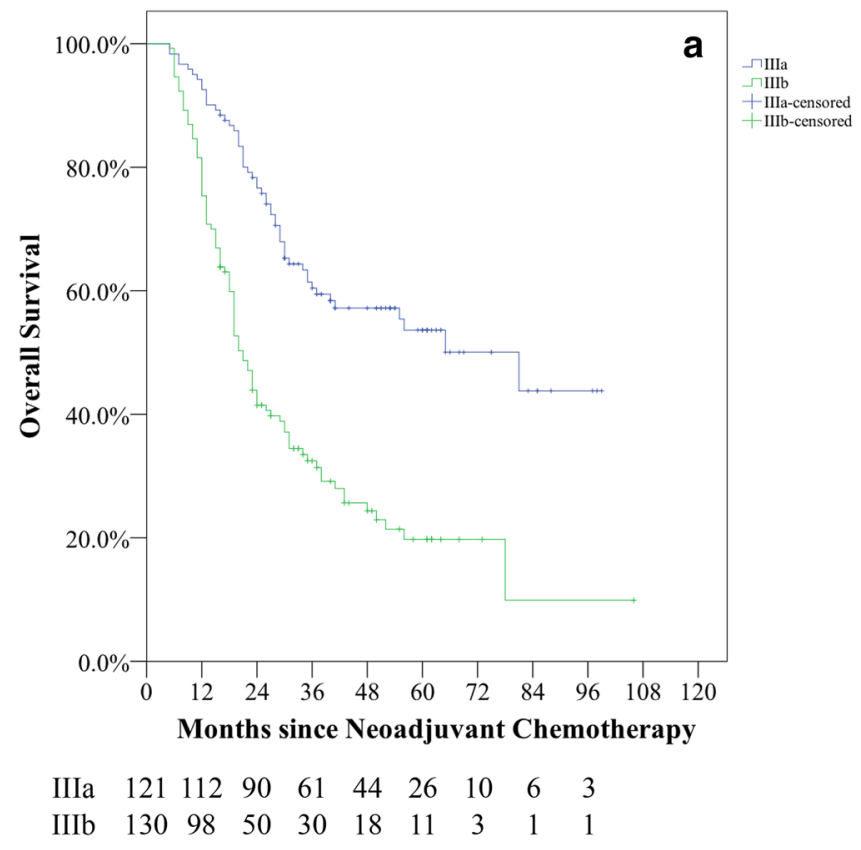

Fig. 3 Subgroups of stage III and survival. Stage III patients were stratified into two subgroups: IIIa group, including 121 patients with stages of ypT4aN1M0, ypT3N2M0, or ypT4aN2M0; IIIb group, including 130 patients with stages of ypT2N3M0, ypT4bN0M0, ypT4bN1M0, ypT3N3M0, ypT4bN2M0, ypT4bN3M0, or ypT4aN3M0. The 3-year overall survival (OS) and disease free survival (DFS)

\section{Discussion}

Neoadjuvant therapy has been widely employed in the treatment of patients with gastric cancer. The eighth edition of TNM Staging Manual describes the staging system of patients with neoadjuvant therapy for the first time, which is undoubtedly a huge step forward, and has set unified standards and a solid foundation for patient evaluation following neoadjuvant chemotherapy. Furthermore, the manual used data from both Eastern and Western countries to revise the pTNM staging system. These intercontinental collaborations may improve the generalizability and applicability of the tumor staging system to different regions and ethnicities, as there have been discrepancies between the East and West regarding the staging and treatment of gastric cancer $[1,3$, 5]. However, the ypTNM staging system included in the eighth edition was solely based on data obtained from the American population. To the best of our knowledge, this is the first study assessing the prognostic value of the ypTNM system in the Asian population.

We found that both ypT and ypN were independent risk factors for disease prognosis. However, ypT could not differentiate the patient survival. One potential explanation for this phenomenon may be that ypT can represent postneoadjuvant tumor status, but not the tumor response to neoadjuvant chemotherapy, which may not be adequate to

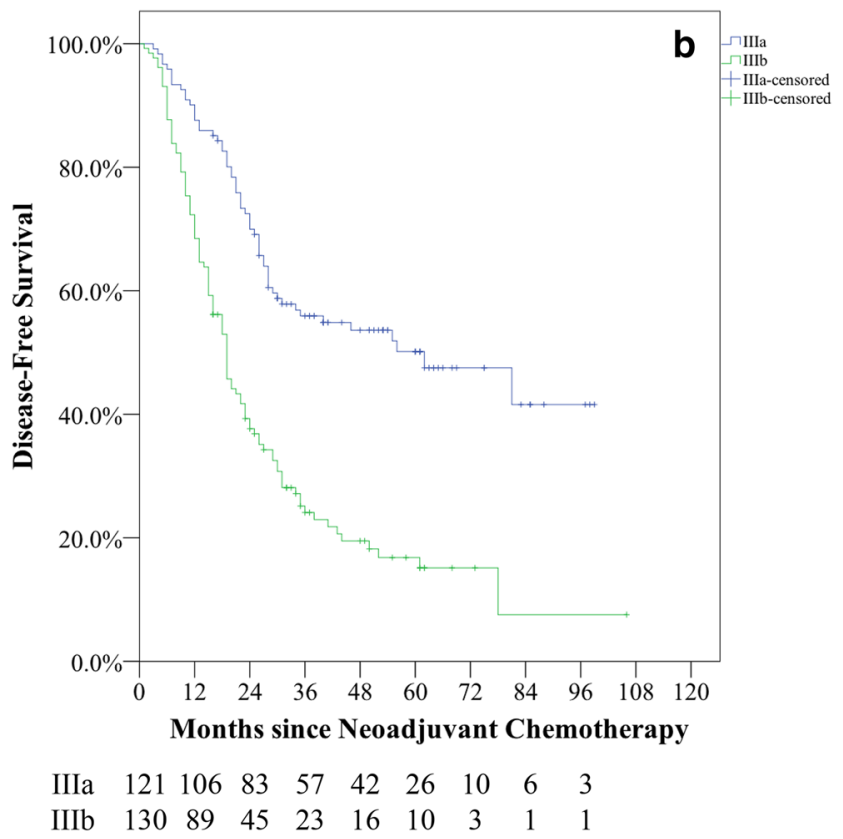

were 60.4 and $55.9 \%$ in the IIIa group, and 32.5 and $24.1 \%$ in the IIIb group; the 5-year OS and DFS were 53.6 and $50.2 \%$ in the IIIa group, and 19.8 and $16.8 \%$ in the IIIb group. Survival analysis found significant worse survival IIIb patients than the IIIa ones with regard to OS (a HR 2.615, 95\% CI 1.862-3.673, $p<0.001$ ) and DFS (b HR 2.616, 95\% CI 1.888-3.624, $p<0.001)$, respectively

account for patient prognosis. Pathologic regression grade is one indicator to evaluate the efficacy of neoadjuvant chemotherapy. However, patients with high ypT stages do not necessarily respond poorly to chemotherapy. For instance, studies have found that up to $50 \%$ of patients in stage ypT3 and ypT4 can have residual tumor cells of less than $10 \%$, whereas the percentage of residual tumor cells was not necessarily smaller than $10 \%$ in those patients with stage ypT1 [12]. Therefore, a few studies have proposed that the ypN stage, combined with pathologic regression grade, may be helpful in stratifying the prognosis of esophageal cancer patients who undergo preoperative chemotherapy or chemoradiotherapy $[6,13]$. This proposition may also hold true for gastric cancer. Despite the significance of chemotherapy-induced tumor response to prognosis prediction, it has not been included in any of current cancer staging systems. This is partly due to the limited accuracy in evaluating malignancies with the current radiological and endoscopic examination strategies, which is a shortcoming acknowledged in the latest AJCC guidelines [3]. Nonetheless, one should note that our explanation is mainly speculation and the underlying mechanism of this phenomenon is yet to be determined. Further analysis in this regard should be encouraged.

The eighth edition of the TNM Staging Manual did not provide any subgrouping standards for ypTNM [3]. Based 
Table 2 Univariate analysis of overall survival and disease free survival $(N=442)$

\begin{tabular}{|c|c|c|c|c|}
\hline & \multicolumn{2}{|l|}{ Overall survival } & \multicolumn{2}{|l|}{ Disease free survival } \\
\hline & HR $(95 \%$ CI $)$ & $p$ & HR $(95 \%$ CI $)$ & $p$ \\
\hline Age & $1.02(1-1.03)$ & 0.028 & $1.01(1-1.03)$ & 0.047 \\
\hline Gender & $0.94(0.66-1.33)$ & 0.72 & $0.97(0.69-1.35)$ & 0.84 \\
\hline Family history & $0.76(0.52-1.12)$ & 0.17 & $0.7(0.48-1.03)$ & 0.07 \\
\hline BMI & & 0.003 & & 0.011 \\
\hline$<18.5$ & Referent & & Referent & \\
\hline $18.5-25.0$ & $0.58(0.36-0.93)$ & 0.024 & $0.62(0.38-1)$ & 0.05 \\
\hline $25.0-30.0$ & $0.39(0.23-0.68)$ & 0.001 & $0.42(0.24-0.71)$ & 0.001 \\
\hline$\geq 30.0$ & $0.19(0.05-0.83)$ & 0.027 & $0.41(0.14-1.2)$ & 0.10 \\
\hline ECOG score & & 0.13 & & 0.25 \\
\hline 0 & Referent & & Referent & \\
\hline 1 & $1.34(1-1.8)$ & 0.05 & $1.27(0.96-1.7)$ & 0.10 \\
\hline 2 & $1.41(0.74-2.7)$ & 0.30 & $1.23(0.65-2.35)$ & 0.53 \\
\hline ASA score & & 0.61 & & 0.49 \\
\hline 1 & Referent & & Referent & \\
\hline 2 & $1.08(0.71-1.64)$ & 0.71 & $1.03(0.69-1.54)$ & 0.88 \\
\hline 3 & $1.27(0.77-2.11)$ & 0.35 & $1.27(0.78-2.07)$ & 0.33 \\
\hline Tumor location & & $<0.001$ & & $<0.001$ \\
\hline Upper & Referent & & Referent & \\
\hline Middle & $1.17(0.75-1.83)$ & 0.48 & $1.21(0.79-1.84)$ & 0.38 \\
\hline Lower & $0.9(0.65-1.26)$ & 0.56 & $0.91(0.66-1.26)$ & 0.57 \\
\hline Whole & $3.23(2.04-5.14)$ & $<0.001$ & $3.09(1.99-4.8)$ & $<0.001$ \\
\hline Diameter in short axis & $1.16(1.11-1.22)$ & $<0.001$ & $1.16(1.11-1.21)$ & $<0.001$ \\
\hline Diameter in long axis & $1.12(1.08-1.16)$ & $<0.001$ & $1.11(1.07-1.15)$ & $<0.001$ \\
\hline Pathological type & & $<0.001$ & & $<0.001$ \\
\hline Adenocarcinoma or adenocarcinoma with NET & Referent & & Referent & \\
\hline $\begin{array}{l}\text { Signet ring cell carcinoma or adenocarcinoma with } \\
\text { signet ring }\end{array}$ & $2.53(1.81-3.55)$ & $<0.001$ & $2.49(1.8-3.44)$ & $<0.001$ \\
\hline Mucinous adenocarcinoma* & $0.9(0.47-1.63)$ & 0.74 & $1.04(0.6-1.81)$ & 0.89 \\
\hline Differentiate grade & & 0.14 & & 0.10 \\
\hline High & Referent & & Referent & \\
\hline Middle & $0.58(0.34-0.99)$ & 0.046 & $0.57(0.34-0.97)$ & 0.037 \\
\hline Low & $0.73(0.46-1.15)$ & 0.17 & $0.75(0.48-1.18)$ & 0.21 \\
\hline Vascular cancer embolus & $0.4(0.3-0.53)$ & $<0.001$ & $0.37(0.28-0.49)$ & $<0.001$ \\
\hline ypT stage & & $<0.001$ & & $<0.001$ \\
\hline $\mathrm{T} 1$ & Referent & & Referent & \\
\hline $\mathrm{T} 2$ & $1.89(0.7-5.1)$ & 0.21 & $1.69(0.68-4.24)$ & 0.26 \\
\hline $\mathrm{T} 3$ & $3.33(1.27-8.7)$ & 0.014 & $3.06(1.26-7.39)$ & 0.013 \\
\hline $\mathrm{T} 4 \mathrm{a}$ & $3.57(1.46-8.73)$ & 0.005 & $3.23(1.42-7.33)$ & 0.005 \\
\hline $\mathrm{T} 4 \mathrm{~b}$ & $13.34(5.11-34.82)$ & $<0.001$ & $10.32(4.27-24.99)$ & $<0.001$ \\
\hline ypN stage & & $<0.001$ & & $<0.001$ \\
\hline N0 & Referent & & Referent & \\
\hline N1 & $1.41(0.86-2.31)$ & 0.18 & $1.53(0.96-2.45)$ & 0.07 \\
\hline $\mathrm{N} 2$ & $3.06(1.97-4.75)$ & $<0.001$ & $2.98(1.95-4.56)$ & $<0.001$ \\
\hline N3 & $5.28(3.53-7.91)$ & $<0.001$ & $5.51(3.74-8.13)$ & $<0.001$ \\
\hline Pathological stage & & $<0.001$ & & $<0.001$ \\
\hline 0 & Referent & & Referent & \\
\hline I & $2.85(1.27-6.41)$ & 0.011 & $2.67(1.25-5.71)$ & 0.011 \\
\hline II & $7.68(3.6-16.41)$ & $<0.001$ & $7.4(3.64-15.07)$ & $<0.001$ \\
\hline
\end{tabular}


Table 2 (continued)

\begin{tabular}{|c|c|c|c|c|}
\hline & \multicolumn{2}{|l|}{ Overall survival } & \multicolumn{2}{|c|}{ Disease free survival } \\
\hline & HR $(95 \%$ CI $)$ & $p$ & HR $(95 \%$ CI $)$ & $p$ \\
\hline III & $1(0.99-1.01)$ & 0.82 & $1(0.99-1.01)$ & 0.82 \\
\hline Postoperative stay & $1(1-1.01)$ & $<0.001$ & $1(1-1.01)$ & $<0.001$ \\
\hline Surgery duration & $1(1-1)$ & 0.80 & $1(1-1)$ & 0.86 \\
\hline Blood loss & $1.47(0.65-3.32)$ & 0.35 & $1.47(0.69-3.12)$ & 0.32 \\
\hline Gastrectomy type & & $<0.001$ & & $<0.001$ \\
\hline Total & Referent & & Referent & \\
\hline Distal & $0.49(0.36-0.69)$ & $<0.001$ & $0.52(0.38-0.71)$ & $<0.001$ \\
\hline Proximal & $0.52(0.31-0.86)$ & 0.011 & $0.49(0.30-0.81)$ & 0.005 \\
\hline Thoratic-abdominal joint & $1.89(1.02-3.52)$ & 0.044 & $2.18(1.23-3.87)$ & 0.008 \\
\hline Multi-organ excision (yes) & $1.79(1.16-2.77)$ & 0.009 & $1.7(1.12-2.58)$ & 0.013 \\
\hline Number of resected LN & $1.01(1-1.02)$ & 0.13 & $1.01(1-1.02)$ & 0.12 \\
\hline Number of metastatic LN & $1.06(1.05-1.07)$ & $<0.001$ & $1.05(1.04-1.07)$ & $<0.001$ \\
\hline
\end{tabular}

$B M I$ body mass index, ASA American Society of Anesthesiologists, ECOG Eastern Clinical Oncology Group, Mucinous adenocarcinoma include mucinous adenocarcinoma, adenocarcinoma with mucinous adenocarcinoma, and mucinous adenocarcinoma with signet ring, $L N$ lymph node

Table 3 Multivariate analysis of overall survival and disease free survival $(N=442)$

\begin{tabular}{|c|c|c|c|c|}
\hline \multirow[t]{2}{*}{ Factors } & \multicolumn{2}{|l|}{ Overall survival } & \multicolumn{2}{|c|}{ Disease free survival } \\
\hline & $\operatorname{HR}(95 \% \mathrm{CI})$ & $p$ & $\mathrm{HR}(95 \% \mathrm{CI})$ & $p$ \\
\hline Age & $1.02(1.01-1.04)$ & 0.011 & $1.02(1-1.03)$ & 0.011 \\
\hline BMI & & 0.027 & & \\
\hline$<18.5$ & Referent & & & \\
\hline $18.5-25.0$ & $0.6(0.36-1)$ & 0.05 & & \\
\hline $25.0-30.0$ & $0.45(0.25-0.81)$ & 0.008 & & \\
\hline$\geq 30.0$ & $0.21(0.05-0.91)$ & 0.038 & & \\
\hline Diameter in short axis & $1.09(1.02-1.16)$ & 0.006 & $1.08(1.02-1.14)$ & 0.01 \\
\hline Pathological type & & $<0.001$ & & $<0.001$ \\
\hline Adenocarcinoma or adenocarcinoma with NET & Referent & & Referent & \\
\hline $\begin{array}{l}\text { Signet ring cell carcinoma or adenocarcinoma } \\
\text { with signet ring }\end{array}$ & $2.01(1.4-2.88)$ & $<0.001$ & $1.97(1.4-2.77)$ & $<0.001$ \\
\hline Mucinous adenocarcinoma* & $0.42(0.22-0.81)$ & 0.01 & $0.51(0.28-0.94)$ & 0.03 \\
\hline ypT stage & & $<0.001$ & & $<0.001$ \\
\hline $\mathrm{T} 1$ & Referent & & Referent & \\
\hline $\mathrm{T} 2$ & $1.44(0.53-3.93)$ & 0.48 & $1.51(0.6-3.8)$ & 0.38 \\
\hline T3 & $1.36(0.5-3.67)$ & 0.55 & $1.3(0.52-3.22)$ & 0.58 \\
\hline $\mathrm{T} 4 \mathrm{a}$ & $1.67(0.66-4.25)$ & 0.28 & $1.6(0.69-3.75)$ & 0.28 \\
\hline $\mathrm{T} 4 \mathrm{~b}$ & $5.62(2.06-15.36)$ & 0.001 & $4.24(1.68-10.65)$ & 0.002 \\
\hline ypN stage & & $<0.001$ & & $<0.001$ \\
\hline No & Referent & & Referent & \\
\hline N1 & $1.25(0.75-2.07)$ & 0.4 & $1.4(0.87-2.24)$ & 0.17 \\
\hline $\mathrm{N} 2$ & $2.75(1.73-4.38)$ & $<0.001$ & $2.63(1.68-4.11)$ & $<0.001$ \\
\hline N3 & $3.86(2.51-5.94)$ & $<0.001$ & $4.08(2.69-6.2)$ & $<0.001$ \\
\hline Gastrectomy type & & 0.013 & & 0.008 \\
\hline Total & Referent & & Referent & \\
\hline Distal & $0.63(0.44-0.89)$ & 0.01 & $0.69(0.49-0.97)$ & 0.031 \\
\hline Proximal & $0.89(0.53-1.49)$ & 0.64 & $0.81(0.48-1.36)$ & 0.42 \\
\hline Thoratic-abdominal joint & $1.63(0.86-3.1)$ & 0.14 & $1.92(1.06-3.47)$ & 0.031 \\
\hline
\end{tabular}

$B M I$ Body mass index 
on our cluster analysis, we divided stage III patients into two subgroups, placing ypT4b and ypN3 into stage IIIb and the others into stage IIIa. The modified system had a significant better $c$-index than the current system. Another possible modification on current ypTNM would be to categorize ypT4b and ypN3 patients as stage IV. A revision like this is not unprecedented, as a similar change was made to stage $\mathrm{IV}$ in the eighth edition manual due to the poor prognosis of cT4b patients. However, we did not include stage IV cases, so we are unable to address this question. Nonetheless, our results do suggest the possibility of further subgrouping the current ypTNM system, which may achieve improved prognosis discrimination, which will assist physicians in treatment decision-making.

The new ypTNM staging system does not address pCR and ypT0N1 patients. In our study, there are 31 patients with ypT0 stage and the $5.9 \%$ pCR rate was comparable to the data from previous studies (3-15\%) [14-20]. We found that the DFS and OS of pCR patients were similar to the ypStage I patients. Furthermore, the discrimination ability of the current ypTNM staging system improved when pCR patients were incorporated as stage I. Hence, it may be reasonable to treat patients as stage I, though larger scale analyses are needed to further inform the proper strategy for treating $\mathrm{pCR}$ patients.

There are several limitations embedded in this study. First, our study only included the resectable cases and did not contain stage IV patients. This limited our ability to examine the prognostic implication of ypTNM in patients with stage IV disease. Second, though our sample size is close to that used in the eighth edition manual, more studies with larger sample sizes are needed to evaluate and validate the new ypTNM staging system. Lastly, ypTNM staging is just one of many prognostic staging systems, and the disease outcomes are influenced by various prognostic factors, including the treatment regimen, targeted therapy, radiotherapy, postoperative adjuvant treatment regimen, and patient tolerance and sensitivity to chemotherapy. In this study, we only focused on the neoadjuvant chemotherapy to explore the clinical applications of ypTNM staging. We are still in the process of exploring their values through on-going clinical trials focusing on the other prognostic factors [21-25].

\section{Conclusions}

The eighth edition of the AJCC staging guidelines for gastric cancer provides unified standards for patient evaluation after neoadjuvant chemotherapy. In this retrospective study, we analyzed our database in accordance with the latest staging system with a special focus on the ypTNM staging system. We found significant survival differences between patients of different stages, which suggested that the generalized ypTNM staging system is effective for staging gastric cancers in the Asian population. We also found a better prognostic value of ypN compared with ypT stage and significantly worsened survival outcomes in ypT4b or ypN3 cases. Our data suggest that further analyses may facilitate subgrouping of each stage to reach a more accurate evaluation of prognosis.

Funding This study is supported by Beijing Municipal Science and Technology Commission nos. Z151100004015070, D141100000414002, D141100000414004.

\section{Compliance with ethical standards}

Conflict of interest The authors declare that they have no conflict of interest.

Research involving Human Participants and/or Animals Informed consent The Ethics Committee of Peking University Cancer Hospital approved this study. All patients provided written informed consent. This study does not involve animal study.

Open Access This article is distributed under the terms of the Creative Commons Attribution 4.0 International License (http://creativeco mmons.org/licenses/by/4.0/), which permits unrestricted use, distribution, and reproduction in any medium, provided you give appropriate credit to the original author(s) and the source, provide a link to the Creative Commons license, and indicate if changes were made.

\section{References}

1. Ajani JA, D’Amico TA, Almhanna K, et al. Gastric Cancer, Version 3.2016, NCCN Clinical Practice Guidelines in Oncology. J Natl Compr Cancer Netw. 2016;14(10):1286.

2. Sano T, Coit DG, Kim HH, et al. Proposal of a new stage grouping of gastric cancer for TNM classification: international Gastric Cancer Association staging project. Gastric Cancer. 2017;20(2):217-25.

3. Amin MBES., Greene FL, et al. AJCC cancer staging manual [M], 8th ed. Springer, New York. 2016.

4. Japanese Gastric Cancer A. Japanese classification of gastric carcinoma: 3rd English edition. Gastric Cancer. 2011;14(2):101-12.

5. Waddell T, Verheij M, Allum W, et al. Gastric cancer: ESMOESSO-ESTRO Clinical Practice Guidelines for diagnosis, treatment and follow-up. Ann Oncol. 2013;24(Suppl 6):vi57-63.

6. Schneider PM, Baldus SE, Metzger R, et al. Histomorphologic tumor regression and lymph node metastases determine prognosis following neoadjuvant radiochemotherapy for esophageal cancer: implications for response classification. Ann Surg. 2005;242(5):684-92.

7. Rizk NP, Venkatraman E, Bains MS, et al. American Joint Committee on Cancer staging system does not accurately predict survival in patients receiving multimodality therapy for esophageal adenocarcinoma. J Clin Oncol. 2007;25(5):507-12.

8. Wilke H, Preusser P, Fink U, et al. Preoperative chemotherapy in locally advanced and nonresectable gastric cancer: a phase II study with etoposide, doxorubicin, and cisplatin. J Clin Oncol. 1989;7(9):1318-26. 
9. Cunningham D, Allum WH, Stenning SP, et al. Perioperative chemotherapy versus surgery alone for resectable gastroesophageal cancer. N Engl J Med. 2006;355(1):11-20.

10. Mandard AM, Dalibard F, Mandard JC, et al. Pathologic assessment of tumor regression after preoperative chemoradiotherapy of esophageal carcinoma. Clinicopathologic correlations. Cancer. 1994;73(11):2680-6.

11. Becker K, Langer R, Reim D, et al. Significance of histopathological tumor regression after neoadjuvant chemotherapy in gastric adenocarcinomas: a summary of 480 cases. Ann Surg. 2011;253(5):934-9.

12. Ott $\mathrm{K}$, Blank $\mathrm{S}$, Becker $\mathrm{K}$, et al. Factors predicting prognosis and recurrence in patients with esophago-gastric adenocarcinoma and histopathological response with less than $10 \%$ residual tumor. Langenbeck's Arch Surg. 2013;398(2):239-49.

13. Smyth EC, Fassan M, Cunningham D, et al. Effect of pathologic tumor response and nodal status on survival in the medical research council adjuvant gastric infusional chemotherapy trial. J Clin Oncol. 2016;34(23):2721-7.

14. Leichman L, Silberman H, Leichman CG, et al. Preoperative systemic chemotherapy followed by adjuvant postoperative intraperitoneal therapy for gastric cancer: a University of Southern California pilot program. J Clin Oncol. 1992;10(12):1933-42.

15. Persiani R, D'Ugo D, Rausei S, et al. Prognostic indicators in locally advanced gastric cancer (LAGC) treated with preoperative chemotherapy and D2-gastrectomy. J Surg Oncol. 2005;89(4):227-36 (discussion 237-228).

16. Heger $\mathrm{U}, \mathrm{Blank} \mathrm{S}$, Wiecha $\mathrm{C}$, et al. Is preoperative chemotherapy followed by surgery the appropriate treatment for signet ring cell containing adenocarcinomas of the esophagogastric junction and stomach? Ann Surg Oncol. 2014;21(5):1739-48.

17. Peixoto RD, Cheung WY, Lim HJ. Perioperative chemotherapy for gastroesophageal cancer in British Columbia: a multicentre experience. Curr Oncol (Toronto Ont). 2014;21(2):77-83.
18. Lorenzen S, Thuss-Patience P, Al-Batran SE, et al. Impact of pathologic complete response on disease-free survival in patients with esophagogastric adenocarcinoma receiving preoperative docetaxel-based chemotherapy. Ann Oncol. 2013;24(8):2068-73.

19. Koh YW, Park YS, Ryu MH, et al. Postoperative nodal status and diffuse-type histology are independent prognostic factors in resectable advanced gastric carcinomas after preoperative chemotherapy. Am J Surg Pathol. 2013;37(7):1022-9.

20. Lowy AM, Mansfield PF, Leach SD, Pazdur R, Dumas P, Ajani JA. Response to neoadjuvant chemotherapy best predicts survival after curative resection of gastric cancer. Ann Surg. 1999;229(3):303-8.

21. Tang L, Sun Y, Li Z, et al. Correlation of diffusion weighted MR imaging with the prognosis of local advanced gastric carcinoma after neoadjuvant chemotherapy. Chin J Gastrointest Surg. 2015;18(3):221-6.

22. Li Z, Xing X, Shan F, et al. ABCC2-24C> T polymorphism is associated with the response to platinum $/ 5$-Fu-based neoadjuvant chemotherapy and better clinical outcomes in advanced gastric cancer patients. Oncotarget. 2016;7(34):55449-57.

23. Wu A, Jia Y, Dong B, et al. Apoptosis and KI 67 index correlate with preoperative chemotherapy efficacy and better predict the survival of gastric cancer patients with combined therapy. Cancer Chemother Pharmacol. 2014;73(5):885-93.

24. Li ZY, Shan F, Zhang LH, et al. Complications after radical gastrectomy following FOLFOX7 neoadjuvant chemotherapy for gastric cancer. World J Surg Oncol. 2011;9:110.

25. Li Z, Shan F, Wang Y, et al. Laparoscopic versus open distal gastrectomy for locally advanced gastric cancer after neoadjuvant chemotherapy: safety and short-term oncologic results. Surg Endosc. 2016;30(10):4265-71. 